\title{
Estudo do Reprocessamento de Polietileno de Baixa Densidade (PEBD) Reciclado do Processamento de Extrusão de Filmes Tubulares
}

\author{
Vinicius Pistor, Amauri Chiesa, Ademir J. Zattera \\ Laboratório de Polímeros, UCS
}

\begin{abstract}
Resumo: O mercado de reciclagem para os polímeros termoplásticos encontra-se atualmente em acentuada ascensão. Os materiais reciclados são vistos como materiais de propriedades inferiores em relação ao material virgem. O presente trabalho avaliou as características viscoelásticas e térmicas do polietileno de baixa densidade (PEBD) reciclado. As amostras foram reprocessadas até dez vezes em condições extremas de processamento $\left(300^{\circ} \mathrm{C} / 80 \mathrm{rpm}\right)$ em uma extrusora monorosca, a fim de avaliar modificações estruturais nas suas propriedades. Foram realizadas análises de reometria oscilatória de placas paralelas e calorimetria exploratória de varredura (DSC). A partir das análises de reometria oscilatória foram calculados espectros de relaxação e retardação pelo programa de regularização não-linear (NLREG) e através das análises de DSC no primeiro aquecimento foram calculados os parâmetros cinéticos pelos métodos de Avrami e Freeman-Carroll. Os resultados do estudo reológico demonstraram que as amostras reprocessadas acima de quatro vezes apresentaram aumento da viscosidade complexa e dos módulos de armazenamento e perda, além de fenômenos de relaxação e retardação mais largos. Entretanto, os termogramas de DSC e os parâmetros cinéticos de fusão demonstraram que o PEBD estudado manteve sua estabilidade térmica, independentemente da modificação de suas características viscoelásticas.
\end{abstract}

Palavras-chave: PEBD, reciclagem, reprocessamento, viscoelasticidade, cinética.

\section{Study of the Reprocessing of Low Density Polyethylene (LDPE) Recycled from Extruded Blown Films}

Abstract: The recycling market of thermoplastic polymers is currently in a sharp increase. Recycled materials are regarded as having inferior properties compared to the virgin material. Here we investigated the viscoelastic and thermal properties of the recycled low density polyethylene (LDPE). The samples were reprocessed up to ten times under extreme processing conditions $\left(300{ }^{\circ} \mathrm{C} / 80 \mathrm{rpm}\right)$ in a single screw extruder in order to evaluate structural changes in their properties. Analyses were performed in an oscillatory rheometer with parallel plates and in a differential scanning calorimeter (DSC). From the analysis of the oscillatory rheometry, relaxation and retardation spectra were obtained upon applying a non-linear adjustment using a computational program (NLREG), while the kinetics parameters were estimated from the results of the first DSC heating using the Avrami and Freeman-Carroll methods. From the rheological study we inferred that the samples reprocessed more than four times had an increase in complex viscosity, storage and loss moduli, also displaying wider relaxation and retardation phenomena. However, the DSC thermograms and the melt kinetic parameters showed that the LDPE studied maintained their thermal stability, regardless of the modification in their viscoelastic properties.

Keyworks: $L D P E$, recycling, reprocessing, viscoelasticity, kinetic.

\section{Introdução}

Os polietilenos (PE) são materiais commodities amplamente utilizados. Para a reciclagem destes polímeros, torna-se necessário o entendimento das condições de processamento, reprocessamento e a conservação das suas propriedades. Entretanto, as condições de processamento podem influenciar na orientação das cadeias e nas suas características finais ${ }^{[1]}$, bem como na reutilização do material reciclado.

Dentre os diversos PE industriais, destaca-se o polietileno de baixa densidade (PEBD). Este apresenta propriedades reológicas únicas em comparação aos PE lineares e os de alta densidade, devido as suas longas cadeias altamente ramificadas ${ }^{[2]}$.

As numerosas ramificações encontradas no LDPE influenciam na redução da cristalinidade, se comparado com o polietileno de alta densidade. Isto resulta em um produto flexível com baixo ponto de fusão. Assim, ramificações longas podem conferir características desejáveis de processamento e também uma viscosidade relativamente baixa ${ }^{[3,4]}$

Segundo McLeish ${ }^{[5]}$, as propriedades reológicas dos polímeros fundidos sofrem grande influência do peso molecular, do tamanho das ramificações e topologia. Ao considerar estas variáveis, nota-se que o comportamento reológico dos materiais poliméricos no estado fundido gera uma relação chave entre estrutura, propriedades e processamento ${ }^{[6]}$.

Peacock ${ }^{[7]}$ descreve que a morfologia do polietileno e, portanto, a forma de itens fabricados a partir dele, não é estável quando é submetido a tensão prolongada. Desta forma, a instabilidade morfológica pode se manifestar como fluência, relaxação, fissuras, fratura frágil e quebra sob tensão ambiental. Todas essas variáveis para relaxação em longo prazo envolvem a recomposição gradual das moléculas, por toda amostra, como na fluência e na relaxação da tensão.

No estudo da fluência, podem-se considerar dois tipos de comportamento: a viscoelasticidade linear e não linear. Estas afetam diretamente a resposta de deformação, bem como os fenômenos de relaxação dos materiais. Para a obtenção destes resultados, Brostow e Corneliussen ${ }^{[8]}$, ressaltam que o módulo de relaxação pode ser obtido em todos os níveis de deformação através de um experimento em baixas amplitudes de deformação, regime este denominado viscoelástico linear.

Porém, funções viscoelásticas que envolvem experimentos extremamente longos não são possíveis de serem avaliados. Por isso dal Castel et al. ${ }^{[9]}$, descrevem que funções viscoelásticas como

Autor para correspondência: Ademir J. Zattera, Laboratório de Polímeros, Universidade de Caxias do Sul, Rua Francisco Getúlio Vargas, 1130 
os espectro de relaxação $(H(\tau))$ e retardação $(L(\tau))$ não podem ser medidos, mas somente calculados. Os autores citam também que tais funções são capazes de descrever efeitos que não são possíveis de analisar por outros métodos reológicos.

Corrêa da Silva et al. ${ }^{[10]}$ estudaram estas funções viscoelásticas para o polietileno de baixa densidade (LDPE). Os autores avaliaram consecutivos processamentos em extrusora de monorosca e, a partir de análises de reometria oscilatória, obtiveram resultados de $(\mathrm{H}(\tau))$ e $(L(\tau))$. Eles concluíram que o uso das funções viscoelásticas descreve com mais clareza o comportamento reológico de polímeros submetidos a consecutivos processamentos.

Delgaldillo-Velázquez et al. ${ }^{[1]}$, avaliaram o comportamento termo-reológico de uma série de misturas de polietileno linear de baixa densidade e polietilenos de baixa densidade (LLDPE/LDPE), também utilizando funções de relaxação. Os autores encontraram concordâncias entre os métodos utilizados e observaram também um forte efeito das ramificações nas propriedades reológicas, gerado pelo PEBD nas misturas. Também é conhecida na literatura a utilização das funções viscoelásticas, por exemplo, em estudo da miscibilidade de polietilenos no estado fundido ${ }^{[12]}$ e na influência da presença de nanocargas em matrizes poliolefínicas ${ }^{[6]}$. Além das propriedades viscoelásticas, a determinação de parâmetros cinéticos pode ser útil no entendimento das modificações cristalinas impostas pelo processamento. Os métodos de Avrami e Freeman-Carrol (F-C) foram utilizados neste trabalho.

As derivações originais realizadas por Avrami ${ }^{[13-15]}$ foram simplificadas por Evans ${ }^{[16]}$ e colocadas no contexto dos polímeros por Meares ${ }^{[17]}$ e Hay ${ }^{[18]}$; este método possibilita a obtenção de resultados como a constante de velocidade $k^{\prime}$, o expoente de Avrami $n$, que possibilita o entendimento do controle de nucleação e crescimento dos cristais na cristalização, e o tempo de meia vida $t_{1 / 2}$, que é o tempo necessário para que $50 \%$ da reação/fenômeno ocorra. O método proposto por Freeman-Carrol possibilita a obtenção dos valores de energia aparente de ativação $\left(E_{a}\right)^{[19]}$. Neste trabalho, foram estudadas as propriedades reológicas e cinéticas do polietileno de baixa densidade (LDPE) reciclado, em condições extremas de processamento, com intuito de avaliar a estabilidade deste material.

\section{Experimental}

\section{Materiais}

Neste estudo foram utilizadas aparas de polietileno de baixa densidade (PEBD; TX 9003, petroquímica Triunfo), oriundo da fabricação de filmes plásticos, gerados pela Empresa Bazei Plásticos e Embalagens Ltda. Este material é adequado para a produção de filmes tubulares. Mesmo havendo filmes fabricados a partir de misturas de polietilenos, os filmes de PE fabricados pela empresa que forneceu as aparas são essencialmente compostos pelo PEBD TX 9003.

\section{Processamento}

As aparas de polietileno foram reprocessadas, em uma extrusora MIOTTO com rosca de diâmetro igual a $90 \mathrm{~mm}, \mathrm{~L} / \mathrm{D} 25$, com corte no cabeçote submerso, troca telas hidráulico, e sistema de alimentação forçada. O fluxo da água do corte no cabeçote foi mantido em $40 \mathrm{~m}^{3} / \mathrm{h}$ a $75^{\circ} \mathrm{C}$, a troca de telas (filtro) foi realizada no início de cada teste, utilizando-se telas malha Reps com 80/110 mesh. A velocidade da rosca foi de $80 \mathrm{rpm}$, e o perfil de temperatura esta representado na Tabela 1.

As amostras foram reprocessadas até 10 vezes nas condições apresentadas na Tabela 1. Desta forma, a nomenclatura assumida para a classificação das amostras foi, por exemplo, $300{ }^{\circ} \mathrm{C} / 80 \mathrm{rpm} \times 2$, sendo a temperatura máxima na zona 5 , a velocidade da rosca e a quantidade de vezes que foi reprocessado, respectivamente.

\section{Reometria oscilatória}

Foram realizadas análises de reometria oscilatória para as amostras em um reômetro oscilatório Anton Paar Physica MCR 101, com placas paralelas de $25 \mathrm{~mm}$ de diâmetro e espaçamento entre as placas de $1 \mathrm{~mm}$, temperatura de ensaio de $200{ }^{\circ} \mathrm{C}$, faixa de frequência de 0,1 à $100 \mathrm{~Hz}$, tensão máxima de ensaio de $500 \mathrm{~Pa}$ e fluxo de nitrogênio de $1 \mathrm{~m}^{3} / \mathrm{h}$. A partir destas análises reológicas foram obtidos os espectros de relaxação $(\mathrm{H}(\tau))$ e retardação $(\mathrm{L}(\tau))$ pelo programa de regularização não-linear (NLREG) $)^{[20]}$.

\section{Calorimetria exploratória diferencial (DSC)}

As análises de DSC (DSC50 - Shimadzu) foram realizadas em atmosfera de nitrogênio ( $50 \mathrm{~mL} / \mathrm{min})$, utilizando-se aproximadamente $10 \mathrm{mg}$ de cada amostra. As amostras foram aquecidas de 25 até $220^{\circ} \mathrm{C}$ em taxa de aquecimento de $10^{\circ} \mathrm{C} / \mathrm{min}$.

\section{Parâmetros viscoelásticos}

Sabe-se que através de um experimento em baixas amplitudes de deformação, o módulo de relaxação pode ser obtido em todos os níveis de deformação ${ }^{[8]}$. No entanto, diversas funções físicas, como o espectro de relaxação $H(\tau)$, não são possíveis de serem calculadas diretamente através de experimentos. Todavia, essas funções podem ser obtidas a partir de uma quantidade experimentalmente mensurável através da resolução de um problema inverso ${ }^{[21]}$.

Através do uso da regularização de Tikhonov em sua generalização não-linear para problemas inversos, denominado método de regularização não-linear, e implementado no programa NLREG, é possível obter funções como o espectro de relaxação $(\mathrm{H}(\tau))$ e retardação $(\mathrm{L}(\tau))^{[20]}$.

Estas funções viscoelásticas podem ser obtidas através dos módulos de armazenamento $\left(G^{\prime}\right)$ e módulo de perda $\left(G^{\prime \prime}\right)$ pelas Equações 1 e 2:

$$
\begin{aligned}
& G^{\prime}(\omega)=G_{\theta}+\int_{-\infty}^{\infty} H(\tau) \frac{\omega^{2} \tau^{2}}{1+\omega^{2} \tau^{2}} d \ln \tau \\
& G^{\prime \prime}(\omega)=\int_{-\infty}^{\infty} H(\tau) \frac{\omega \tau}{1+\omega^{2} \tau^{2}} d \ln \tau
\end{aligned}
$$

onde: $G_{\theta}$ é o módulo no equilíbrio, $\mathrm{H}(\tau)$ a função do espectro de relaxação e $\tau$ é o tempo de relaxação ou retardação.

$\mathrm{O}$ espectro de relaxação é resultado da soma de infinitos elementos de Maxwell que descrevem o comportamento mecânico da porção elástica do polímero, bem como sua capacidade de armazenar energia $^{[20]}$.

A estimativa do espectro de retardação é realizada através das inter-relações dos espectros descrita por Ferry, e resulta do somatório de infinitos elementos de Voigt que são responsáveis por toda a porção viscosa do polímero ${ }^{[22]}$ (Equações 3 e 4):

$$
\begin{aligned}
& H(\tau)=\frac{L(\tau)}{\left[J_{g}+\int_{-\infty}^{\infty} \frac{L(\tau)}{(1-\tau) / \tau} d \ln \tau-\frac{\tau}{\eta_{0}}\right]^{2}+\pi^{2} L(\tau)^{2}} \\
& L(\tau)=\frac{L(\tau)}{\left[G_{\theta}+\int_{-\infty}^{\infty} \frac{H(\tau)}{\tau /(1-\tau)} d \ln \tau\right]^{2}+\pi^{2} L(\tau)^{2}}
\end{aligned}
$$

onde $J_{g}$ é a compliância instantânea e $\eta_{0}$ a viscosidade na taxa zero. 


\section{Parâmetros cinéticos}

Para o modelo de Avrami ${ }^{[13-15]}$, em condições isotérmicas, a determinação do grau de conversão $\alpha(t)$ segue a Equação 2 (Equação 5).

$$
1-\alpha(t)=\exp \left(-k t^{n}\right)
$$

onde $k$ é a constante de Avrami, que depende da taxa de nucleação, e $n$ é o expoente de Avrami, dependente do modelo de nucleação. Linearizando a equação de Avrami pode-se obter a Equação 6:

$$
\ln [-\ln (1-\alpha(t))]=\ln k+n \ln t
$$

Desta forma, isolando os termos $\ln [-\ln (1-\alpha(t))] v s$. ln $t$ em um gráfico, uma relação linear é observada, e é possível obter $k$ e $n$ a partir do coeficiente linear e angular, respectivamente.

Jeziorny ${ }^{[23]}$, considerando condições não-isotérmicas na aplicação do modelo de Avrami, determinou uma relação entre $k$ e a taxa de aquecimento $\varphi$ para valores de $k^{\prime}$ corrigidos (Equação 7).

$$
\ln k^{\prime}=\frac{\ln k}{\phi}
$$

Ainda pelo modelo de Avrami é possível determinar o tempo de meia vida da reação $\left(t_{1 / 2}\right)$ que é o tempo necessário para que $50 \%$ da reação ou fenômeno ocorram (Equação 8).

$$
t_{1 / 2}=\left(\frac{\ln 2}{k^{\prime}}\right)^{1 / n}
$$

A Equação 9 foi desenvolvida por Freeman e Carroll (F-C) para calcular a energia de ativação $\left(E_{a}\right)^{[19]}$ :

$$
\frac{\Delta \ln (d \alpha / d t)}{\Delta \ln (1-\alpha)}=\left(\frac{E_{a}}{R}\right)\left(\frac{\Delta(1 / T)}{\Delta \ln (1-\alpha)}\right)
$$

onde $\alpha$ é a conversão, $R$ a constante dos gases e $T$ é a temperatura absoluta. Isolando os termos da Equação 1, representados nos eixos da Figura 8, é possível obter valores de $E_{a}$ a partir do coeficiente angular da reta.

\section{Resultados e Discussão}

A Figura 1 apresenta o comportamento da viscosidade complexa $\left(\eta^{*}\right)$ em função da frequência angular $(\omega)$. Como pode ser observado, o LDPE puro e a amostra reprocessada duas vezes, em baixas frequências, demonstraram menor viscosidade em relação às demais amostras reprocessadas. Este comportamento pode ser devido ao consumo dos aditivos antioxidantes, bem como modificação da estrutura molecular. Shanina e Zaikov ${ }^{[24]}$ estudaram o consumo de um antioxidante específico em uma composição de PEBD-PP estabilizada; os autores puderam estimar que, a uma temperatura de $60{ }^{\circ} \mathrm{C}$, a concentração de antioxidantes inicial diminuiria para um valor critico em cerca de quatro anos. Os autores ainda ressaltam que apesar dos valores não serem precisos, podem servir como um ponto de referência no processo de avaliação da vida útil do material. Assim, pode-se supor que a taxa de consumo do antioxidante para as amostras estudadas no presente trabalho seja superior devido às elevadas temperaturas de processamento do PEBD.

Tabela 1. Perfil de temperatura utilizada ao longo do barril da extrusora.

\begin{tabular}{cc}
\hline Parâmetros & Temperaturas $\left({ }^{\circ} \mathbf{C}\right)$ \\
\hline Temperatura da matriz & 190 \\
Temperatura troca telas & 220 \\
Zona 5 & 300 \\
Zona 4 & 250 \\
Zona 3 & 200 \\
Zona 2 & 190 \\
Zona 1 & 170 \\
\hline
\end{tabular}

Segundo Scott e Chakraborty ${ }^{[25]}$, a ausência do antioxidante pode gerar a formação de peróxidos e hidroperóxidos formados a partir de reações via radicais livres, o que poderia justificar o aumento da viscosidade para as amostras reprocessadas quatro e dez vezes, pois a formação de radicais livres poderia ocasionar a formação de ligações cruzadas ao longo das ramificações do PEBD.

A partir das análises de reometria oscilatória, foram obtidos resultados de módulo de armazenamento $\left(G^{\prime}\right)$ e módulo de perda $(G$ ”) que correspondem às respostas elástica e viscosa, respectivamente ${ }^{[12,26]}$. A Figura 2 apresenta os resultados de $G$ ' e $G$ ", para as amostras estudadas.

Os resultados obtidos para $G^{\prime}$ e $G$ " demonstraram comportamento semelhante aos da Figura 1, i.e., em baixas frequências os módulos do LDPE puro e o de duas vezes reprocessado apresentaram menores valores do que aqueles encontrados para as reprocessadas quatro e dez vezes. Estes resultados reforçam as características relacionadas à estrutura do material, onde o aumento dos módulos para as amostras reprocessadas quatro e dez vezes pode ser referente à formação de ligações cruzadas. Porém, segundo Martins et al..$^{[3]}$, a formação de ligações cruzadas, por provocar um aumento no peso

\begin{tabular}{|c|c|}
\hline$\rightarrow-G^{\prime}$ PEBD puro & $\rightarrow \mathrm{G}^{\prime} / 300^{\circ} \mathrm{C} / 80 \mathrm{rpm} \times 2$ \\
\hline$\triangle-\mathrm{G}^{\prime} / 300^{\circ} \mathrm{C} / 80 \mathrm{rpm} \times 4$ & $\rightarrow-\mathrm{G}^{\prime} / 300^{\circ} \mathrm{C} / 80 \mathrm{rpm} \times 10$ \\
\hline -.- G" PEBD puro & ..•. G" $/ 300^{\circ} \mathrm{C} / 80 \mathrm{rpm} \times 2$ \\
\hline ..- G" $/ 300^{\circ} \mathrm{C} / 80 \mathrm{rpm} \times 4$ & .*. G $\mathrm{G}^{\prime \prime} / 300^{\circ} \mathrm{C} / 80 \mathrm{rpm} \times 10$ \\
\hline
\end{tabular}

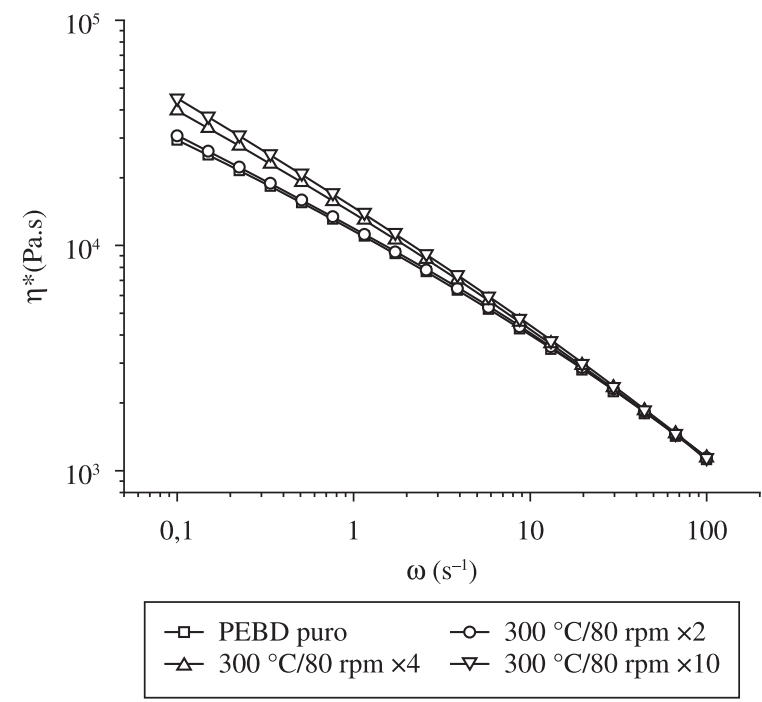

Figura 1. Curvas de viscosidade complexa obtidas através das análises de reometria oscilatória.

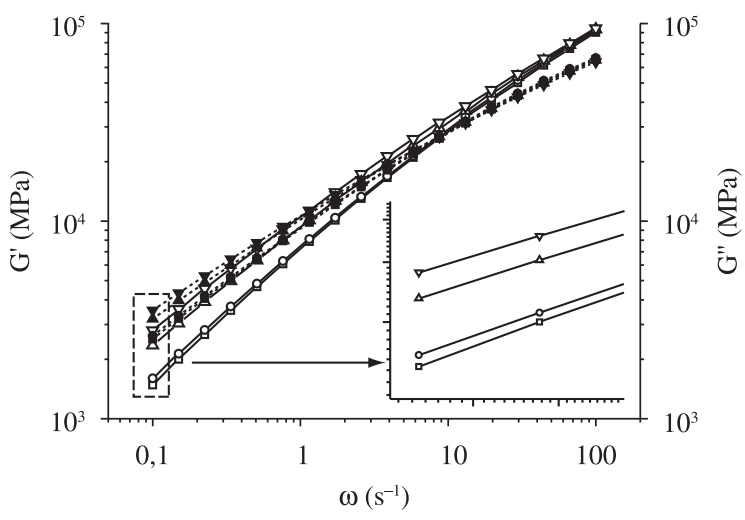

Figura 2. Resultados de módulo de armazenamento $(G$ ') e perda $(G$ ”). 
molecular, pode gerar melhorias das propriedades, ao contrário da cisão das moléculas, o que reduziria o peso molecular resultando na perda das propriedades.

A Figura 3 apresenta as curvas de fluxo obtidas pela relação de Cox-Merz ${ }^{[27]}$. As aproximações de Cox-Merz possibilitam relacionar a viscosidade cinemática e a viscosidade complexa. A obtenção das curvas de fluxo possibilita uma compreensão maior dos fenômenos decorrentes do processamento, uma vez que a viscosidade se encontra como uma função da taxa de cisalhamento. Como pode ser observado, em baixas taxas de cisalhamento são mais evidentes as modificações de viscosidade impostas pelos reprocessamentos. Entretanto, os resultados obtidos são correspondentes com aqueles observados pelas Figuras 1 e 2. A obtenção das curvas de fluxo possibilita também a aplicação da lei das potências ${ }^{10,27]}$ para quantificar o índice de consistência e índice das potências. A Tabela 2 apresenta os resultados obtidos pela lei das potências. Segundo Corrêa da Silva et al. ${ }^{[10]}$, o índice de consistência $(\mathrm{K})$ é o valor da viscosidade onde a taxa de cisalhamento é igual à unidade. Já o índice das potências $\left(\mathrm{n}_{\mathrm{p}}\right)$, segundo Cruz et al. ${ }^{[28]}$, é uma medida da pseudoplasticidade dos polímeros, sendo assim uma consequência do efeito de desemaranhamento, orientação molecular e também da geração de temperatura por cisalhamento. Os autores citam também que quanto mais próximo de zero o valor de $n_{p}$, mais pseudoplástico é o material, sendo que qualquer pequena variação no cisalhamento acarretará em variações de viscosidade, prejudicando o processamento.

Na Tabela 2 pode ser observado que os valores de $\mathrm{K}_{\mathrm{p}}$ aumentaram conforme o material foi sendo reprocessado, aumentado aproximadamente 1,5 vezes o valor de viscosidade do material puro para o material reprocessado dez vezes. Este aumento pode ser devido à formação de ligações cruzadas e aumento da quantidade de ramificações. Segundo Odian ${ }^{[29]}$, o comprimento das ramificações formadas pela transferência de cadeia, no caso deste trabalho através da degradação, afeta diretamente a fluidez e, portanto, as características de processamento.

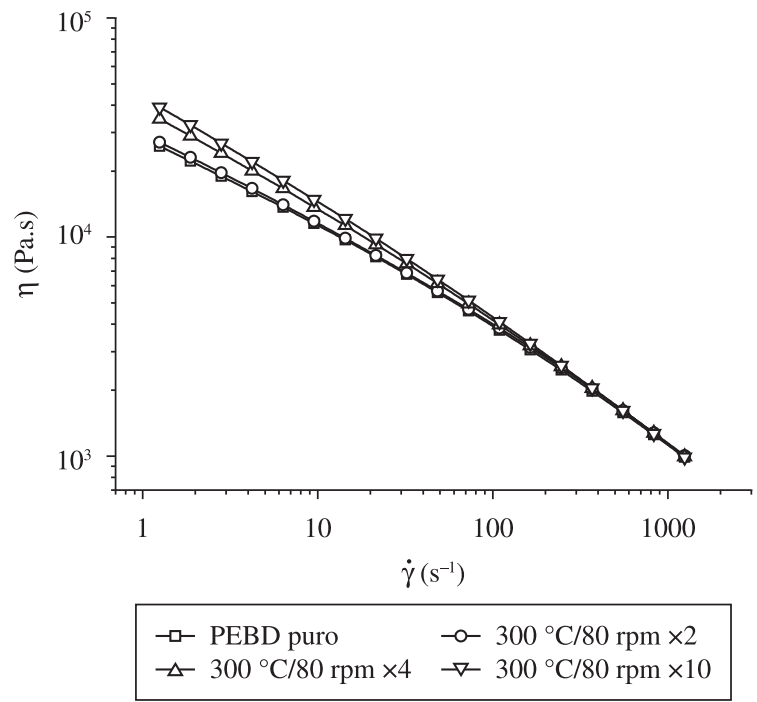

Figura 3. Curvas de fluxo obtidas pela relação de Cox-Merz.

Tabela 2. Parâmetros obtidos através da lei das potências.

\begin{tabular}{ccc}
\hline Amostra & $\mathbf{K}_{\mathbf{p}}($ Pa.s $)$ & $\mathbf{n}_{\mathbf{p}}$ \\
\hline PEBD puro & 27437 & 0,53 \\
$300^{\circ} \mathrm{C} / 80 \mathrm{rpm} \times 2$ & 28359 & 0,53 \\
$300^{\circ} \mathrm{C} / 80 \mathrm{rpm} \times 4$ & 36495 & 0,49 \\
$300^{\circ} \mathrm{C} / 80 \mathrm{rpm} \times 10$ & 41525 & 0,47 \\
\hline
\end{tabular}

Waldman e De Paoli ${ }^{[30]}$ em estudo da degradação termo-mecânica de polipropileno (PP), polietileno de baixa densidade (PEBD) e uma blenda de PP/PEBD através do processamento em reômetro de torque a $230{ }^{\circ} \mathrm{C}$ observaram que o processamento destes materiais acarreta na degradação e formação de ligações cruzadas. Os autores ainda demonstraram que a formação de radicais alquila provenientes da cisão homolítica de ligações $\mathrm{C}-\mathrm{H}$ podem reagir com oxigênio $\left(\mathrm{O}_{2}\right)$ e a reticulação torna-se possível pela formação de ligações peroxídicas entre as cadeias do polímero.

Entretanto, os valores de $n_{p}$ apresentaram tendência de redução, de 0,53 para 0,47 , conforme foi sendo reprocessado, aumentando assim sua pseudoplasticidade. Isto sugere que, independente da possível formação de ligações cruzadas, o material tende a manter características significativamente aptas para o reprocessamento.

Os resultados de $G$ ' e $G$ " foram utilizados para determinar espectros relaxação e retardação característicos das amostras. As Figuras 4 e 5 apresentam os espectros de relaxação $(\mathrm{H}(\tau))$ e retardação $(\mathrm{L}(\tau))$ obtidos pelo método de regularização não linear $\left(\right.$ NLREG) ${ }^{[11]}$. Segundo dal Castel et al..$^{[9]}$, estas funções são capazes de descrever comportamentos imperceptíveis a outros métodos de caracterização.

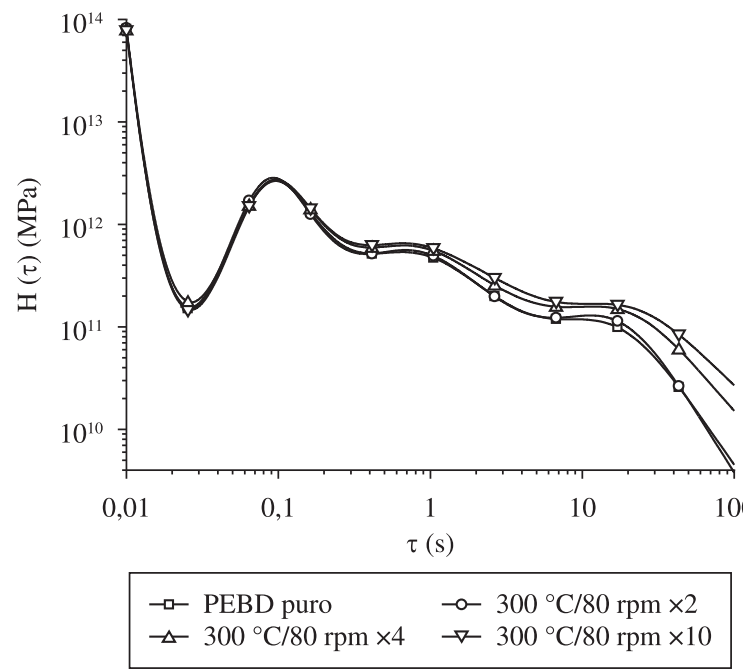

Figura 4. Espectros de relaxação obtidos para as amostras reprocessadas.

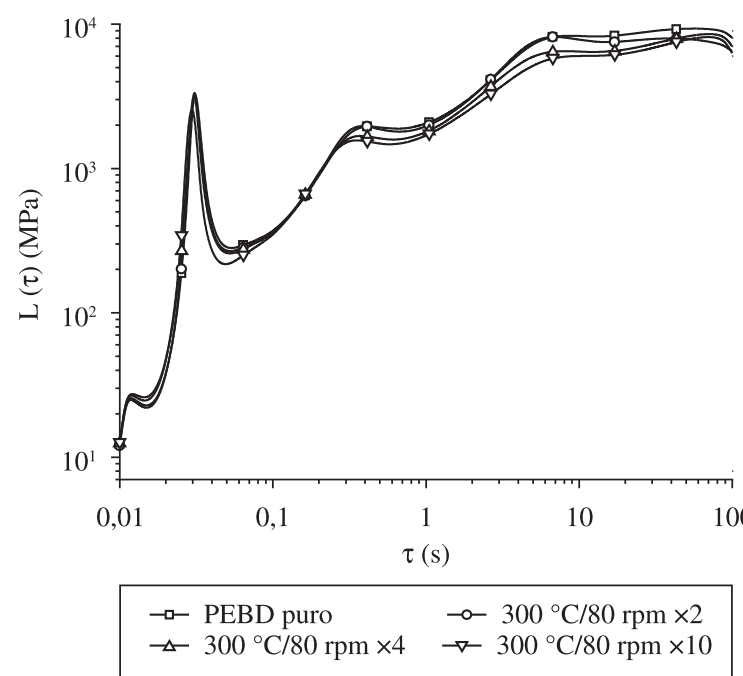

Figura 5. Espectros de retardação obtidos para as amostras reprocessadas. 
As cadeias poliméricas apresentam uma ampla distribuição dos tempos de relaxamento que pode se estender ao longo de várias décadas no tempo ou em domínios de frequência. Às vezes, a resposta é rápida, principalmente elástica, enquanto que às vezes é curta, principalmente viscosa ${ }^{[26]}$.

Analisando os espectros de relaxação da Figura 5, podem ser observados três tempos distintos de relaxação. Os tempos de relaxação observados estão relacionados à estrutura do PEBD; o tempo de relaxação inicial $(\tau \approx 0,1$ segundo) possivelmente seja referente à distribuição do peso molecular, visto que as moléculas menores tendem a relaxar por primeiro. $\mathrm{O}$ segundo fenômeno de relaxação ( $\tau \approx 1$ segundo) pode estar relacionado com o tipo e quantidade de ramificações visto que o impedimento espacial imposto por grandes ramificações seria logicamente um atraso na relaxação em relação às moléculas menores. E, por fim, o terceiro tempo de relaxação $(\tau \approx 10$ segundos) possivelmente esteja associado ao rompimento dos efeitos topológicos como, por exemplo, o entrelaçamento de cadeias longas $^{[32]}$.

Esta ordem de fenômenos sugere que as estruturas relaxam por um efeito cooperativo. Riande et al. ${ }^{[26]}$ sugerem que a cooperatividade ocorre mesmo quando a relaxação se propaga ao longo das cadeias, envolvendo um número crescente de segmentos da cadeia principal com o passar do tempo. Comparando os espectros de relaxação (Figura 4), é possível observar que a partir do material ser reprocessado quatro e dez vezes, os espectros apresentaram tempos

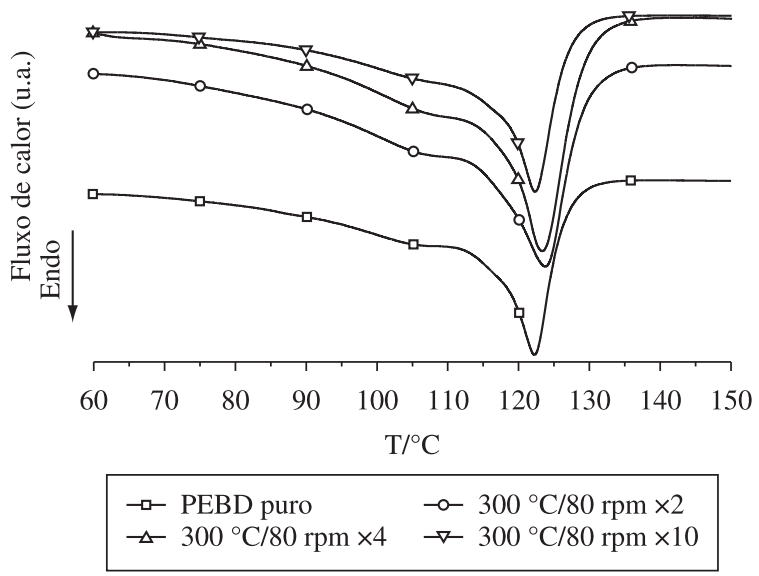

Figura 6. Termogramas de DSC obtidas a taxa de $10{ }^{\circ} \mathrm{C} / \mathrm{min}$.

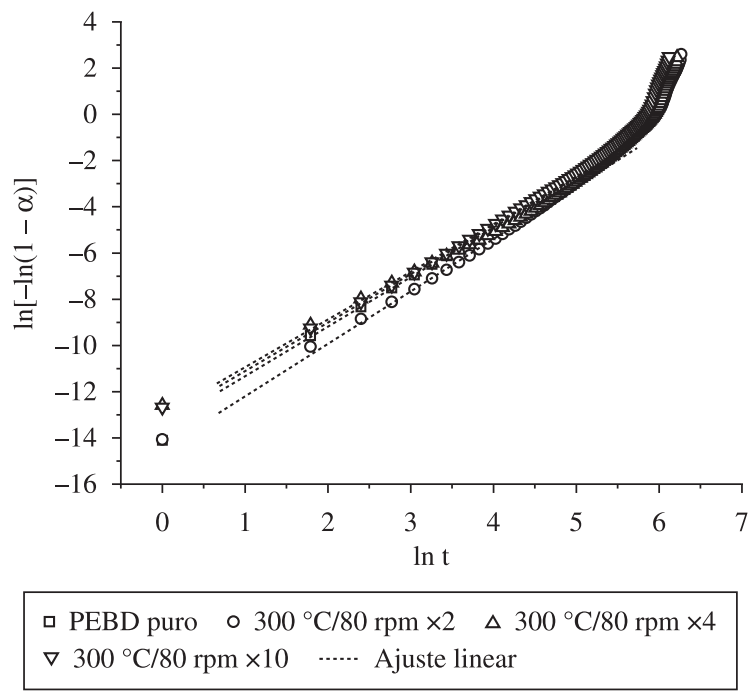

Figura 7. Linearização dos termos da equação de Avrami. de relaxação mais largos em 1 e 10 segundos. Sugestivamente, para 1 segundo como a relaxação é possivelmente associada ao tipo e quantidade de ramificações, os tempos mais largos sugerem que existe maior dificuldade de afastamento das cadeias, logo em 10 segundos associado ao rompimento dos efeitos topológicos por cooperatividade são afetados também, demonstrando um forte indício de que este efeito possa estar relacionado à formação de ligações cruzadas.

Os mesmos efeitos podem ser observados para os espectros de retardação na Figura 5, onde os espectros das amostras reprocessadas quatro e dez vezes apresentam os dois últimos fenômenos de retardação das moléculas mais largos ao longo do tempo, corroborando assim os espectros de relaxação. Desta forma, para compreender o efeito do aumento na viscosidade, módulo e os fenômenos observados para e relaxação e retardação, foram realizadas análises de calorimetria exploratória diferencial, a fim de avaliar o comportamento de fusão no primeiro aquecimento para as amostras estudadas. A Figura 6 apresenta os termogramas de DSC obtidos para o primeiro aquecimento das amostras de PEBD.

Pode ser observado que as amostras apresentam uma transição de primeira ordem em torno de $100-140{ }^{\circ} \mathrm{C}$. Esta larga faixa de fusão é atribuída pela literatura pela também larga distribuição de peso molecular do PEBD, devido as suas ramificações e obviamente pela distribuição e variação dos tamanhos de cristais $^{[7]}$. Como podem ser observadas pela análise de DSC, todas as amostras apresentaram um comportamento de fusão similar, sugerindo assim que o aumento da viscosidade e os efeitos das análises reológicas estejam relacionados somente com algumas regiões de formação de ligações cruzadas, visto que este fenômeno não prejudicou o fenômeno de fusão das amostras.

Entretanto, alguns efeitos relacionados à ação da temperatura na destruição dos cristais podem ser detalhados avaliando alguns parâmetros cinéticos. Sendo assim, a Figura 7 apresenta um gráfico de $\ln [-\ln (1-\alpha)] v s$. ln t para a obtenção dos parâmetros de Avrami. Segundo Liu et al. ${ }^{[31]}$ a equação de Avrami representa apenas as partes iniciais de cristalização dos polímeros corretamente.

Segundo Sperling ${ }^{[27]}$, os esferulitos crescem para fora com uma taxa de crescimento constante radial até que acontece um choque devido ao crescimento terminar na intersecção dos cristais. Esta consideração pode ser tomada também para o processo inverso, a fusão, desta forma a região referente ao ajuste linear para a obtenção dos parâmetros cinéticos foi determinada na região mais linear da Figura 7.

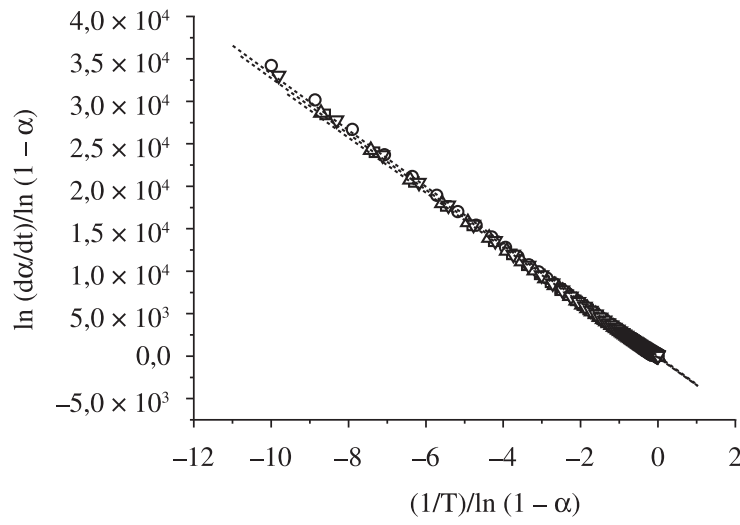

口 PEBD puro $\quad 300^{\circ} \mathrm{C} / 80 \mathrm{rpm} \times 2 \quad \triangle 300^{\circ} \mathrm{C} / 80 \mathrm{rpm} \times 4$ $\nabla 300^{\circ} \mathrm{C} / 80 \mathrm{rpm} \times 10 \quad \cdots \cdot$... Ajuste linear

Figura 8. Linearização dos termos da equação de Freeman-Carroll. 
Tabela 3. Parametros cinéticos obtidos pelos métodos de Avrami e Freeman-Carroll.

\begin{tabular}{|c|c|c|c|c|c|c|}
\hline \multirow[t]{2}{*}{ Amostra } & \multicolumn{4}{|c|}{ Avrami } & \multicolumn{2}{|c|}{ F-C } \\
\hline & $k^{\prime}$ & $t_{1 / 2}($ minutos $)$ & $n$ & $\mathbf{r}$ & $E_{a}\left(\mathrm{~kJ} \cdot \mathrm{mol}^{-1}\right)$ & $\mathbf{r}$ \\
\hline PEBD puro & 0,13 & 2,20 & 2,17 & 0,9999 & 26,80 & 0,9995 \\
\hline $300^{\circ} \mathrm{C} / 80 \mathrm{rpm} \times 2$ & 0,13 & 2,09 & 2,30 & 0,9986 & 27,68 & 0,9995 \\
\hline $300{ }^{\circ} \mathrm{C} / 80 \mathrm{rpm} \times 4$ & 0,12 & 2,35 & 2,01 & 0,9971 & 26,81 & 0,9997 \\
\hline $300^{\circ} \mathrm{C} / 80 \mathrm{rpm} \times 10$ & 0,12 & 2,24 & 2,12 & 0,9995 & 27,27 & 0,9995 \\
\hline
\end{tabular}

A Figura 8 apresenta a linearização do modelo de Freeman-Carroll $(\mathrm{F}-\mathrm{C})^{[19]}$. Como pode ser observado a partir da linearização dos termos da equação (9) na Figura 8, foi possível obter os valores de energia aparente de ativação $\left(E_{a}\right)$ através do coeficiente angular para a fusão das amostras estudadas.

Os resultados obtidos pelo método de Avrami e Freeman-Carroll estão representados na Tabela 3. Como pode ser observado, ao traçar os ajustes lineares para ambos os métodos foi possível obter valores do coeficiente de correlação (r), os quais foram todos próximos da unidade.

Para a análise dos parâmetros de Avrami, foi observado que tanto os valores da constante de velocidade $(k$ '), quanto o tempo de meia vida $\left(t_{1 / 2}\right)$ e o expoente de Avrami $(n)$ obtidos se mantiveram praticamente constantes ao longo dos dez reprocessamentos. O expoente de Avrami $n=2$ sugere que a dissociação dos cristais ocorre de maneira predominantemente em duas dimensões ${ }^{[27]}$, ou seja, o processo de fusão é iniciado entre as regiões cristalinas e amorfas. Também corroborando estes resultados, os valores de $E_{a}$ não demonstraram variações significativas, ficando entre 26,80-27,68 kJ.mol ${ }^{-1}$. Como os valores de $E_{a}$ estão relacionados com a dificuldade de ocorrência de um determinado fenômeno, e sendo que não foram observadas modificações significativas em nenhum dos parâmetros cinéticos estudados, é possível supor que os efeitos observados nos estudos reológicos não são um empecilho na reutilização dos materiais reciclados.

\section{Conclusões}

No presente trabalho foram estudadas as propriedades viscoelásticas e cinéticas do reprocessamento de aparas de filmes derivados do PEBD (TX 9003) reciclado.

A partir dos resultados reológicos foi observado que as amostras reprocessadas acima de quatro vezes apresentaram um aumento dos valores de viscosidade complexa e dos módulos de armazenamento e perda, possivelmente referentes à formação de ligações cruzadas e aumento da quantidade de ramificações em algumas regiões do termoplástico. Entretanto, os valores de índice de potência demonstraram que ao longo dos reprocessamentos o PEBD aumentou sua pseudoplasticidade.

Já para os termogramas de DSC foi observado que todas as amostras conservaram suas características de fusão, o que foi corroborado pelos resultados do modelo de Avrami e seguidamente pelo modelo de Freeman-Carrol, onde, os resultados obtidos evidenciaram que o material preserva suas características de fusão, constante de velocidade $\left(k^{\prime}\right)$, ordem de reação $(n)$, bem como a $E_{a}$ na fusão.

Estes resultados demonstraram que mesmo em uma condição extrema $\left(300{ }^{\circ} \mathrm{C} / 80 \mathrm{rpm}\right)$ e com o aumento da viscosidade acima de quatro reprocessamentos, este material ainda conserva características suficientemente aptas para ser reprocessado e reutilizado.

\section{Agradecimentos}

Os autores agradecem a empresa Americanoplast, pela realização dos reprocessamentos, à empresa Bazei Plásticos e Embalagens pelo fornecimento das aparas de filmes de PEBD, ao professor Rudinei Fiorio, pelas sugestões dadas ao trabalho, ao CNPq e a UCS pelo apoio financeiro concedido ao projeto.

\section{Referências Bibliográficas}

1. Ward, I.; Hadley, D. W. - "Mechanical properties of solid polymers", John Wiley \& Sons, England (1993).

2. Yamaguchia, M.; Takahashib, M. - Polym., 42, p.8663 (2001).

3. Martins, A. F.; Suarez, J. C. M. \& Mano, E. B. - Polímeros, 9, 27 (1999).

4. Guerrini, L. M.; Paulin F. P. I. \& Bretas, R. E. S. - Polímeros, 14, p. 38 (2004).

5. McLeish, T. C. - Curr. Opin. Sol. St. M, 2, p.678 (1997).

6. Azizi, H.; Ghasemi, I. \& Karrabi, M. - Polym. Test., 27, p.548 (2008).

7. Peacock, A. J. - "Handbook of Polyethylene: Structures, Properties, and Applications", Marcel Dekker, New York (2000).

8. Brostow, W. \& Corneliussen, R. D. - "Failure of Plastics", New York, Hanser (1986).

9. Dal Castel, C.; Bianchi, O.; Oviedo, M. A. S.; Liberman, S. A.; Mauler, R. S. \& Oliveira, R. V. B. - Mat. Sci. Eng. C, 29, p.602 (2009).

10. Corrêa da Silva, G. A.; Fiorio, R.; Bianchi, O.; Martins, J. D. N. \& Zattera, A. J. in: Anais do $10^{\circ}$ Congresso Brasileiro de Polímeros, CD ROM, Foz do Iguaçu - PR, out (2009).

11. Delgaldillo-Velázquez, O.; Hatzikiriakos, S. G.; Sentmanat, M. Rheol. Acta, 19, p.47 (2008).

12. Barnes, H. A.; Hutton, J. F. \& Walters, K. - "An Introduction to Rheology", Elsevier, (1989).

13. Avrami, M. - J. Chem. Phys., 7, p.1103 (1939).

14. Avrami, M. - J. Chem. Phys., 8, p.212 (1939).

15. Avrami, M. - J. Chem. Phys., 9, p.177 (1940).

16. Evans, U. R. - Trans. Faraday Soc., 41, p.365 (1945).

17. Meares, P. - “Polymers: Structure and Bulk Properties", Van Nostrand, New York (1965).

18. Hay, J. N. - Br. Polym. J., 3, p.74 (1971).

19. Paika, P. \& Kar, K. K. Mat. - Chem. Phys, 113, p.953 (2009).

20. Roths, T.; Marth, M.; Weese, J. \& Honerkamp, J. - Comput. Phys. Commun., 139, p.279 (2001).

21. Weese, J. - Comput. Phys. Commun., 77, p.429 (1993).

22. Ferry, J. D. - "Viscoelastic Properties of Polymers", John Wiley \& Sons, New York (1980).

23. Jeziorny, A. - Polym., 19, p. 1142 (1978).

24. Shanina, E. L. \& Zaikov, G. E. - Polym. Deg. Stab., 51, p. 219 (1996).

25. Scott, G. \& Chakraborty, K. B. - Eur. Polym. J., 13, p. 731 (1977).

26. Riande, E.; Díaz-Calleja, R.; Prolongo, M. G.; Masegosa, R. M. \& Salom, C. "Polymer Viscoelasticity: Stress and Strain in Pratice",, Marcel Dekker, New York (2000).

27. Sperling, L. H. - "Introduction to Physical Polymer Science", John Wiley \& Sons (2006).

28. Cruz, S. A.; Farah, M.; Zanin, M. \& Bretas, R. E. S. - Polímeros, 18, p.144 (2008).

29. Odian, G. "Principles of Polymerization", John Wiley \& Sons (2004).

30. Waldman, W. R. \& De Paoli, M. A. - Polym. Deg. Stab., 60, p.301 (1998).

31. Liu, W.; Hsiao, B. S. \& Stein, R. S. - Polym. Mater. Sci. Eng., 81, p.363 (1999).

32. Pistor, V.; Fiorio, R.; Zattera, A. J., Polym., 51, p.5165 (2010).

Enviado: $17 / 02 / 10$

Reenviado: $30 / 03 / 10$

Aceito: $26 / 04 / 10$

DOI: $10.1590 / \mathrm{S} 0104-14282010005000045$ 